\title{
Références bibliographiques du dossier « les valeurs dans l'éducation »
}

\section{Bernadette Plumelle}

\section{(2) OpenEdition}

12 Journals

Édition électronique

URL : https://journals.openedition.org/ries/11140

DOI : 10.4000/ries. 11140

ISSN : 2261-4265

Éditeur

France Education international

\section{Édition imprimée}

Date de publication : 1 septembre 2021

Pagination : 173-184

ISBN : 978-2-85420-630-2

ISSN : 1254-4590

Référence électronique

Bernadette Plumelle, "Références bibliographiques du dossier « les valeurs dans l'éducation » », Revue internationale d'éducation de Sèvres [En ligne], 87 | septembre 2021, mis en ligne le 01 septembre 2022, consulté le 24 mars 2023. URL : http://journals.openedition.org/ries/11140 ; DOI : https://doi.org/ 10.4000/ries. 11140 


\title{
Références bibliographiques du dossier « les valeurs dans l'éducation »"
}

\author{
Bernadette Plumelle \\ Ingénieure de recherche (honoraire)
}

L'actualité parfois dramatique de ces dernières années en France a posé de manière aiguë la question des valeurs partagées ou non par les populations, valeurs différentes selon les générations, et de leur transmission par l'école. Mais de quelles valeurs s'agit-il et y a-t-il des conflits de valeurs? L'école est-elle porteuse de valeurs différentes de celles de la société? Pour ce dossier, la bibliographie donne une place particulière à des ouvrages d'ordre théorique qui appartiennent au champ des sciences sociales car la question des valeurs, avant d'être portée par les systèmes éducatifs, est fortement débattue par les sociologues, les philosophes, les historiens... Les références sélectionnées, majoritairement en langue française, sont récentes, afin d'offrir un aperçu actuel des débats particulièrement vifs au sein du monde occidental sur le sujet de la transformation des valeurs dans la société et dans l'éducation. Cette sélection complète les titres proposés par les contributeurs du dossier.

La bibliographie propose deux parties introductives, l'une qui rassemble quelques publications, d'ordre théorique, sur les valeurs, l'autre qui propose les résultats d'enquêtes menées en Europe et ailleurs sur les valeurs revendiquées au sein des sociétés. La section centrale offre des références sur les valeurs de l'éducation et sur l'éducation aux valeurs au regard des évolutions qu'ont connues les sociétés néolibérales. Dans les parties suivantes, il est question des valeurs dans différents horizons culturels, de celles promues par les organisations internationales, puis des " éducations à ». La bibliographie s'achève sur une sélection de références sur les valeurs enseignées en France.

Les résumés sont, pour l'essentiel, ceux des éditeurs et des revues.

Bibliographie arrêtée le 6 juillet 2021.

\section{QU'EST-CE QUE LES VALEURS?}

\begin{abstract}
DELMAS-MARTY Mireille, Vers une communauté de valeurs. Les forces imaginantes du droit (IV), Paris: Seuil, 2011, 448 p.

Comment oser parler de communauté de droit à l'échelle d'une planète livrée aux affrontements, à la violence et à l'intolérance? Et comment concevoir les contours d'une communauté de valeurs par-delà la diversité des cultures et l'opposition des intérêts? Explorant d'une part les interdits fondateurs, d'autre part les droits fondamentaux (droits de l'homme et biens publics mondiaux), l'autrice, dans la perspective d'un humanisme nouveau, pluriel et ouvert, montre que le droit peut contribuer à nourrir l'idée de bien commun.
\end{abstract}


HEINICH Nathalie, Des valeurs. Une approche sociologique, Paris: Gallimard, 2017, $405 p$.

L'autrice montre que les valeurs ne sont ni des réalités ni des illusions, mais des représentations collectives cohérentes et agissantes. La «sociologie axiologique "s'attache à ce que sont les valeurs pour les acteurs: comment ils évaluent, opinent, pétitionnent, expertisent; comment ils attribuent de «la " valeur, en un premier sens, par le prix, le jugement ou l'attachement. L'analyse pragmatique des jugements produits en situation réelle de controverse permet à l'autrice de mettre en évidence la culture des valeurs que partagent les membres d'une même société.

\section{REVAULT D'ALLONNES Myriam, La faiblesse du vrai. Ce que la post-vérité fait à notre monde commun, Paris: Seuil, 2018, 144 p.}

La post-vérité est définie comme ce qui se rapporte «aux circonstances dans lesquelles les faits objectifs ont moins d'influence sur l'opinion publique que ceux qui font appel à l'émotion ou aux croyances personnelles ». Elle brouille la distinction essentielle du vrai et du faux, portant atteinte à la capacité à vivre ensemble dans un monde commun. L'autrice questionne les rapports conflictuels entre politique et vérité; le problème majeur de la politique n'est pas celui de sa conformité à la vérité, c'est son lien à la constitution de l'opinion publique et à l'exercice du jugement.

ROSA Hartmut, Accélération. Une critique sociale du temps, Paris: La Découverte, 2013, $480 p$.

L'auteur, sociologue et philosophe allemand, considère l'accélération comme un trait caractéristique de la modernité dès son origine. L'ouvrage a pour thème central l'accélération sociale du temps, analysée selon trois dimensions: l'innovation technique, le changement social et le rythme de la vie. L'accélération du changement social produit un changement de la société qui affecte la politique, l'art, la science, les relations professionnelles comme la vie domestique et l'éthique. Le rythme du changement culturel et structurel devient supérieur à celui de la succession des générations.

\section{WEBER Max, Le savant et le politique, Paris: La Découverte, 2003, 210 p.}

L'ouvrage contient deux conférences prononcées respectivement par Max Weber en 1917 et 1919, l'une sur «le métier et la vocation de savant ", l'autre sur « le métier et la vocation d'homme politique». Pour étudier ces deux figures, l'auteur conjugue une approche historico-sociologique et une interrogation éthique sur le sens que peuvent avoir l'une et l'autre ainsi que sur les responsabilités qu'elles engagent. Le travail du savant implique une spécialisation rigoureuse afin d'atteindre son but: celui de démontrer la vérité à partir de faits et d'arguments reconnus comme scientifiquement valables. L'acteur politique est un homme d'action qui agit: il prend position en fonction de ses valeurs. 


\section{VALEURS ET SOCIÉTÉS}

BRECHON Pierre, GONTHIER Frédéric, Les valeurs des Européens. Évolutions et clivages, Paris: Armand Colin, 2014, 288 p.

La grande enquête consacrée aux valeurs des Européens montre que les spécificités propres aux pays n'ont pas disparu. Depuis 1981, les différences restent très importantes entre le nord et le sud de l'Europe, tout comme entre l'ouest et l'est. Qu'il s'agisse de la famille, de la sociabilité, de la politique, du travail ou de la religion, la diversité demeure extrêmement forte. La carte des valeurs n'est pas pour autant figée. On constate partout une montée des valeurs d'individualisation, même si leur développement est inégal selon les grandes aires culturelles de l'Europe. L'ouvrage montre les effets du développement économique, de l'élévation du niveau d'études, de la montée de la sécularisation, des mutations de l'État-providence sur la transformation des valeurs.

CICCELLI Vicente, "Valeurs des jeunes ", in VAN ZANTEN Agnès, RAYOU Patrick (sous la direction de), Dictionnaire de l'éducation, Paris: PUF, 2017 (2e édition), p. 853-856

Les valeurs changent avec le temps et témoignent des évolutions des sociétés et des groupes sociaux à l'égard des autres. L'auteur analyse la question des liens entre les générations, la recomposition du rapport à la démocratie et l'existence de valeurs universalistes au sein de la jeunesse.

GALLAND Olivier, "Les générations au prisme des valeurs ", Futurible, février 2021, $n^{\circ}$ 441, p. 51-64 [disponible sur le portail Cairn]

L'auteur examine ce que révèlent les enquêtes sur les valeurs des Européens s'agissant des différences de valeurs entre générations. Regardées sur une longue période, les valeurs exprimées par les Européens ne divergent pas autant qu'on pourrait le penser en fonction de l'âge. Partant de plusieurs grands systèmes de valeurs (libéralisme culturel, traditions et religiosité, implication dans la vie sociale et confiance envers les autres) analysés sur une trentaine d'années, l'auteur montre combien les différences de valeurs sont bien davantage fonction du pays d'appartenance que de l'âge des répondants.

SCHWARTZ Shalom H., "Les valeurs de base de la personne: théorie, mesures et applications ", Revue française de sociologie, 2006, vol. 47, no 4, p. 929-968 [disponible sur le portail Cairn]

Le concept de valeur en sciences sociales a souffert d'une absence de consensus, que ce soit sur la définition des valeurs, sur leur contenu ou sur la structure des relations qu'elles entretiennent les unes avec les autres. Cet article présente des données provenant de plus de soixante-dix pays. On traite ici de dix valeurs de base que les individus reconnaissent comme telles dans toutes les cultures. Les individus, comme les groupes, se distinguent par les priorités qu'ils donnent à ces valeurs. On examine d'où proviennent les différences de priorité que les individus accordent aux valeurs, et les conséquences en termes d'attitudes et de comportements qui découlent de ces différences de priorités. 


\section{LES VALEURS DANS L’ÉDUCATION}

CHAUVIGNÉ Céline, FABRE Michel, HAGÈGE Hélène (coord.), "L'école entre valeurs et savoirs: quel développement critique possible? Dossier", Éducation et socialisation. Les cahiers du CERFEE, juin 2018, $n^{\circ} 48$, n.p. [en ligne]

L'école a toujours éduqué mais les valeurs connaissent aujourd'hui un regain d'intérêt, comme antidotes idéalisés aux crises de la société. Comment à la fois construire du commun et donner aux élèves les repères et les outils intellectuels pour s'orienter dans les problématiques des questions socialement vives et susceptibles de développer l'esprit critique? Comment articuler savoirs et valeurs, dans le cadre de l'école? Les contributeurs questionnent les valeurs, repèrent les différents cadres dans lesquels elles sont formulées et travaillées, et analysent leurs tensions intrinsèques pour proposer des pistes permettant une éducation au discernement. https://journals.openedition.org/edso/2926

\section{DAVIET Barbara, "Repenser le principe d'éducation comme bien public ", Recherche et prospective en éducation, juillet 2016, $n^{\circ} 17,11$ p. [en ligne]}

Après la Seconde Guerre mondiale, l'éducation a été considérée comme un bien public devant être prise en charge par l'État. Depuis, le paysage éducatif a considérablement changé et le rôle de l'État est de plus en plus contesté par les partisans du marché. Ainsi, l'approche humaniste de l'éducation perd du terrain au profit d'un discours économique envisageant l'éducation comme une marchandise. L'auteure analyse le concept de bien public et interroge sa capacité à contrer les effets de la privatisation et de la marchandisation de l'éducation. Elle propose ainsi la notion d'éducation comme bien commun, inscrite dans une perspective humaniste. https://bit.ly/3hFLWcW

DEWEY John, Démocratie et éducation suivi de Expérience et éducation, Paris: Armand Colin, 2011, 446 p.

Ce volume propose les deux grands textes de John Dewey sur l'éducation: son traité de pédagogie, Démocratie et éducation (1916) et Expérience et éducation (1938). Comment centrer l'éducation sur « les propres activités sociales de l'enfant » plutôt que sur les savoirs? Comment l'éducation peut-elle préparer l'enfant aux conditions sociales qu'il connaîtra hors de l'école? Dewey place l'expérience au cœur de l'apprentissage et recentre la pédagogie sur l'individu plutôt que sur les savoirs: l'école doit répondre à la curiosité naturelle de l'enfant et lui apprendre à désirer et entreprendre. La finalité de l'école est d'accroître la capacité à agir et c'est en cela qu'elle participe de la démocratie.

\section{DUBET François, La préférence pour l'inégalité. Comprendre la crise des solida-} rités, Paris: Seuil, 2014, 106 p.

Depuis les années 1980, les inégalités se creusent partout en Amérique du Nord et en Europe. Au même moment, on observe un reflux des États-providence. Même si chacun le déplore, l'égalité concrète est de moins en moins désirée. Mais ce ne sont pas seulement les crises et les inégalités qui affectent les liens de solidarité, c'est aussi la faiblesse de ces liens qui explique que les inégalités se creusent. En dépit de leurs principes affichés, les sociétés «choisissent » l'inégalité. Ce livre montre que l'aggravation des inégalités procède d'une crise des solidarités entendues comme l'attachement à des liens sociaux qui nous font désirer l'égalité de tous, y compris de ceux que nous ne connaissons pas. 
DURKHEIM Émile, L'éducation morale, cours de sociologie fait à la Sorbonne en 1902-1903; édition électronique réalisée par Jean-Marie Tremblay à partir de l'édition Librairie Félix Alcan (Paris, 1934) [en ligne]

Ce document reproduit le premier cours sur la science de l'éducation que donna Durkheim à la Sorbonne. Il comporte dix-huit leçons regroupées en trois parties. Dans la première partie intitulée "Les éléments de la moralité ", il distingue les éléments constitutifs de la morale laïque: l'esprit de discipline, l'attachement aux groupes sociaux, l'autonomie de la volonté. https://bit.ly/2V93WVj

\section{FABRE Michel, GOHIER Christiane (sous la direction de), Les valeurs éducatives au risque du néo-libéralisme, Presses universitaires de Rouen et du Havre, 2015, 168 p.}

Cet ouvrage réunit des contributions sur les valeurs de l'éducation en rapport avec les politiques néo-libérales. La première partie "Questions d'école » interroge les mutations qu’a connues l'école au regard de l'idéologie néo-libérale. Les auteurs de la deuxième partie s'appuient sur des penseurs (Dewey, Honneth, Foucault, etc.) pour cerner les caractéristiques du néo-libéralisme. Dans la troisième partie, les auteurs posent un certain nombre de diagnostics sur les confusions de valeurs.

\section{FABRE Michel, FRELAT-KAHN Brigitte, PACHOD André (sous la direction de), L'idée} de valeur en éducation. Sens, usages, pertinence, Paris: Hermann, 2016, 288 p.

Le langage de la valeur fait aujourd'hui question. Quels peuvent être les enjeux contemporains d'une philosophie des valeurs dans le contexte éducatif? Quelles sont les valeurs éducatives convoquées dans les discours sur l'éducation aujourd'hui? Sur quels modes le sont-elles? Et quelle peut être la valeur de ces valeurs? Une première partie explore diverses acceptions et déterminations que peut avoir la notion de valeur. Une deuxième partie interroge la pertinence du discours sur les valeurs dans le contexte d'une société sécularisée, plurielle et portée au relativisme. Comment l'éducation peut-elle encore se rapporter aux valeurs? Les analyses se centrent, dans la troisième partie, sur des valeurs particulières en vue d'élucider leur signification et les conditions de leur transmission, dans les milieux éducatifs et particulièrement à l'école.

GALICHET François, "L'émancipation par le savoir: à quelles conditions?", Recherches en éducation, 2018, $n^{\circ} 34$, n.p. [en ligne]

La philosophie des Lumières considérait le savoir comme un moyen d'émancipation. L'époque moderne fait du savoir une fin: il serait émancipateur car il donne de la société une connaissance vraie qui est émancipatrice en elle-même et par elle-même. Dans les deux cas se trouve occultée la question de l'appropriation du savoir, autrement dit de la pédagogie. Dans la mesure où savoir et rapport au savoir ne sont pas dissociables, les modalités pédagogiques de sa transmission ne sont plus secondaires; selon les choix qu'elles opèrent, elles seront ou non émancipatrices. https://journals.openedition.org/ree/1827

HOUSSAYE Jean, "Valeurs du système d'enseignement", in VAN ZANTEN Agnès, RAYOU Patrick (sous la direction de), Dictionnaire de l'éducation, Paris: PUF, 2017 (2édition), p. 856-861

L'école est un monde de valeurs et les débats qui la traversent sont de l'ordre des valeurs. Les valeurs structurent et habitent l'école. L'auteur s'attache à en dessiner les lignes de force en trois parties: valeurs et éducation; valeurs, éducation et société; valeurs, pluralisme et pédagogie. 


\section{NUSSBAUM Martha C., Not for profit: why democracy needs the humanities, Oxford: Princeton University Press, 2010, 158 p.}

Martha Nussbaum, philosophe américaine, présente un plaidoyer pour une certaine vision de l'éducation et des humanités en s'appuyant sur ses travaux universitaires consacrés au rôle des émotions, de l'imagination et de la narration dans la vie morale et politique. L'éducation tournée vers le profit économique ("education for profit») cherche avant tout à doter les élèves d'un certain nombre de capacités techniques pour évoluer dans un monde de concurrence économique mondialisée, contrairement au modèle d'une éducation tournée vers la démocratie (" education for democracy») car l'éducation exclusivement tournée vers le profit sape lentement les conditions qui permettent aux sociétés démocratiques de fonctionner.

PRAIRAT Eirick, "La valeur en débats ", in FABRE Michel, FRELAT-KAHN Brigitte, PACHOD André (sous la direction de), L'idée de valeur en éducation. Sens, usages, pertinence, Paris: Hermann, 2016, p. 61-77

L'auteur fait le point sur les grands débats philosophiques menés ces dernières décennies autour de la question de la valeur et de ses enjeux en philosophie de l'éducation. La deuxième partie examine trois débats qui animent la philosophie de l'éducation: la question de la valeur de l'enseignement, la question des valeurs à promouvoir et transmettre; la question des valeurs requises pour enseigner. La troisième partie analyse le sens et les enjeux de l'éthique professionnelle et de la transmission des valeurs.

\section{SANDEL Michael, La tyrannie du mérite, Paris: Albin Michel, 2021, 384 p.}

L'auteur, professeur à Harvard, se livre ici à une critique de l'élite du diplôme. Donner le pouvoir aux plus compétents plutôt qu'aux mieux nés est apparu, après la Seconde Guerre mondiale, comme un objectif juste et rationnel. Comment la méritocratie s'est-elle muée en «tyrannie du mérite»? Le capital culturel se transmet et la mobilité sociale recherchée n'existe pas vraiment malgré les politiques de discrimination positive (affirmative action). Le fossé entre l'élite surdiplômée et la masse des autres, les médiocrement qualifiés, s'est creusé de manière très inquiétante pour la stabilité des démocraties. À trop valoriser la responsabilité personnelle dans le succès, on dévalorise l'échec, présenté uniquement comme une faute morale.

STRAUME Ingerid S., "The subject and the world: Educational challenges", Educational Philosophy and Theory, 2015, vol. 47, n' 13-14, p. 1465-1476

L'article explore la notion de "sujet», dans le contexte de l'éducation, comme une alternative à des concepts plus limités comme celui d'élève ou d'apprenant. S'inspirant de la pensée de Cornelius Castoriadis, le sujet est un sujet conscient et autoréflexif qui s'organise et se modifie par rapport à un monde de significations qui n'est disponible que dans une société qui s'est instituée de manière explicite ou autonome. L'objectif de cet article est double: d'une part, introduire des réflexions pédagogiques dans les discussions sur la nature humaine et non humaine et, d'autre part, développer un répertoire conceptuel plus riche que celui qui est immédiatement disponible pour explorer les phénomènes éducatifs. 


\section{LES VALEURS PRÔNÉES \\ PAR LES ORGANISATIONS INTERNATIONALES}

Commission européenne, EACEA: Agence executive: éducation, audiovisuel et culture, Eurydice, Promoting citizenship and the common values of freedom tolerance and non-discrimination through education: Overview of education policy developments in Europe following the Paris Declaration of 17 march 2015, Luxembourg: Office des publications de l'Union européenne, mars 2016, 16 p. [en ligne]

Les ministres de l'Union européenne ont adopté la déclaration sur la promotion de l'éducation à la citoyenneté et aux valeurs communes de liberté, de tolérance et de non-discrimination (Déclaration de Paris, 17 mars 2015). Ce rapport fournit un aperçu des évolutions des politiques d'éducation à la citoyenneté et aux valeurs de liberté, de tolérance et de non-discrimination dans les 28 États signataires de la Déclaration de Paris. Ces politiques sont variées et comprennent des stratégies nationales, des plans d'action, de nouvelles réglementations. Elles mettent l'accent sur l'acquisition de compétences sociales, civiques, et interculturelles. https://bit.ly/2Uu7PEd

CONSEIL DE L'EUROPE, Cadre de référence des compétences pour une culture de la démocratie. Volume I: Contexte, concepts et modèle, Strasbourg: Éditions du Conseil de l'Europe, 2019, 87 p. [en ligne]

Le Conseil de l'Europe a élaboré un cadre de référence des compétences nécessaires à une culture de la démocratie, à utiliser dans les curriculums nationaux et les programmes d'enseignement, du primaire au supérieur et en formation professionnelle. Ce volume présente le modèle des compétences nécessaires à une culture de la démocratie, adopté par les ministres européens de l'éducation en avril 2016. https://bit.ly/3yqKvpx

TAWIL Sobhi (coord.), Repenser l'éducation. Vers un bien commun mondial?, Paris: UNESCO, 2015, 95 p. [en ligne]

L'éducation a vocation à préparer les individus et les communautés aux tensions générées par les changements qui surviennent dans le monde, en les rendant capables de s'y adapter et d'y répondre. L'auteur contribue à relancer la réflexion sur l'éducation et l'apprentissage dans ce contexte. Il traite du développement durable, de l'élaboration de politiques éducatives nationales et internationales et réaffirme une approche humaniste de l'éducation. Il considère aussi nécessaire de replacer l'éducation et le savoir dans le contexte de biens communs mondiaux. https://bit.ly/3dS1vNE

UNESCO, Éducation à la citoyenneté mondiale. Préparer les apprenants aux défis $\boldsymbol{d} \boldsymbol{u} \mathbf{X X \boldsymbol { I } ^ { e }}$ siècle, Paris: UNESCO, 2015, 47 p. [en ligne]

Qu'est-ce que l'éducation à la citoyenneté mondiale (ECM) ? Comment l'introduire dans les programmes scolaires? Ce rapport vise à améliorer la compréhension de l'ECM et de ses implications sur les contenus éducatifs et les méthodes d'enseignement dans le monde. Il présente un cadre conceptuel qui comprend les connaissances, les compétences, les valeurs et les comportements nécessaires pour la promotion de la tolérance, de l'équité et de la paix dans un monde plus sûr. L'ECM invite à poser un nouveau regard sur l'éducation, considérée comme un facteur de compréhension et de résolution des problèmes mondiaux d'ordre social, politique, culturel, économique et environnemental. https://bit.ly/3yvwpmC 


\section{L’ÉdUCATION AUX VALEURS}

AUDIGIER François, "Les Éducation à... et la formation au monde social", Recherches en didactiques, 2012, $n^{\circ}$ 14, p. 47-63 [disponible sur le portail Cairn]

Depuis plus d'un siècle, l'école s'efforce de combiner la formation du citoyen et celle du travailleur. Cela implique notamment la transmission de connaissances sur le monde social. Cette formation s'appuie d'une part sur des disciplines comme l'histoire, la géographie, l'éducation à la citoyenneté, d'autre part sur des savoirs parfois rangés dans cette dernière, parfois dans d'autres disciplines, manifestant la difficulté de les définir et de les stabiliser. La priorité accordée aujourd'hui à l'employabilité et les demandes croissantes de nouveaux objets de formation, regroupés sous le terme d'" éducation à », construisent un contexte nouveau qui invite à réfléchir aux contenus et conditions de possibles recompositions disciplinaires pour former au monde social.

BARTHES Angela, LANGE Jean-Marc, TUTIAUX-GUILLON Nicole (sous la direction de), Dictionnaire critique des enjeux et concepts des "éducation à ", Paris: L'Harmattan, 2017, 622 p.

Depuis le début des années 1980, de nombreuses formes d'«éducations à " (la santé, la citoyenneté, la solidarité internationale, les médias et l'information, etc.) sont apparues. Elles s'inscrivent dans le contexte de la mondialisation et résultent des préconisations des instances internationales comme l'Unesco. Ces "éducations à " se situent à la fois dans la continuité des actions éducatives scolaires et en rupture avec cette tradition, car elles ne se cantonnent plus au seul ordre scolaire. Elles s'inscrivent dans un contexte mondialisé caractérisé par de fortes pressions économiques sur le monde éducatif.

Collectif, "Le retour de l'éducation morale. Dossier», Éducation et sociétés, 2018, $n^{o} 42$, p. 5-134

Le projet de l'éducation morale se caractérise par la capacité à gérer des identités plurielles et à interroger l'ordre cognitif ordinaire pour ouvrir l'espace des possibles. La construction de ces compétences repose à la fois sur la culture fournie par les enseignements disciplinaires et sur la diversité des expériences vécues à l'école et hors de l'école. La première partie décline la diversification des conceptions du bien commun et examine la manière dont elles sont mises en œuvre par les politiques éducatives. Une deuxième section aborde le problème de la mise en œuvre. Le développement des compétences civiques doit s'ancrer dans l'expérience des élèves qui peuvent souffrir à l'école de l'injustice ou de l'humiliation. La troisième section traite de l'articulation entre ces préoccupations morales et la nouvelle organisation du travail.

\section{LOVAT Terence, TOOMEY Ron, NEVILLE Clement (eds), International research handbook on values education and student wellbeing, Dordrecht : Springer, 2016, 103 p.}

S'appuyant sur les recherches les plus récentes menées dans le monde entier, ainsi que sur des exemples de bonnes pratiques, l'ouvrage analyse l'éducation aux valeurs dans le contexte d'une série de mesures scolaires associées au bien-être des élèves. Il s'agit notamment du développement social, émotionnel, moral et spirituel. Cette publication intervient au moment où l'éducation aux valeurs élargit son champ d'action, passant de la morale, de l'éthique, de l'instruction civique et de l'éducation à la citoyenneté à une définition plus large, synonyme d'une approche holistique de l'éducation en général. Ce champ d'application élargi est souvent décrit comme une pédagogie liée aux valeurs et au bien-être, en accord avec les recherches récentes en neurosciences. 
OPERTTI Renato, Dix clés pour repenser le curriculum, Genève: UNESCO. Bureau international d'éducation, 2021, 26 p. [en ligne]

Ce document souligne l'urgence de repenser les curriculums au regard de la réaffirmation des engagements de l'Agenda 2030, avec les bouleversements et les changements sociétaux systémiques partout dans le monde, et surtout en fonction de la transformation profonde de l'éducation ainsi que des systèmes éducatifs à l'ère post-Covid-19. L'auteur propose dix clés pour approfondir la compréhension systémique et holistique du curriculum. La clé 6 , intitulée "Promouvoir les synergies entre les valeurs", indique que le programme éducatif 2030 de l'Unesco «pose les bases de l'harmonie, du bien-être, de la justice et du développement durable. Les valeurs constituent le fondement incontournable et bénéfique de tout enseignement et apprentissage, quels que soient sa forme, son contenu ou ses implications ». https://bit.ly/3hln9Fd

\section{TIBBITTS Felisa, Élaboration et révision des programmes d'enseignement pour l'éducation à la citoyenneté démocratique et aux droits de l'homme, Paris: UNESCO, Strasbourg: Conseil de l'Europe, 2016, 112 p. [en ligne]}

L'éducation à la citoyenneté démocratique (ECD) et l'éducation aux droits de l'homme (EDH) jouent un rôle essentiel dans l'édification de sociétés pacifiques, durables et inclusives, fondées sur le respect des droits fondamentaux des individus. Le renforcement des politiques éducatives dans le domaine de l'éducation à la citoyenneté démocratique et de l'éducation aux droits de l'homme est au cœur de l'action du Conseil de l'Europe, de l'Unesco, de l'Organisation pour la sécurité et la coopération en Europe et de l'Organisation des États américains. La publication a été préparée conjointement par ces organisations, afin de soutenir l'engagement des États à promouvoir l'éducation à la citoyenneté et aux droits de l'homme et à améliorer l'accès de tous à une éducation de qualité. Y sont proposées des études de cas (Australie, Colombie, Finlande et Afrique du Sud). https://bit.ly/2SS1jXd

VERHOEVEN Marie, JADOT Évelyne, "Enquête sur l'éducation à la citoyenneté en Belgique francophone: nouvel imaginaire politique et traductions locales fragmentées ", Éducation et sociétés, 2018, $n^{\circ}$ 42, p. 85-100

S'appuyant sur les résultats d'une recherche menée en Belgique francophone, l'article examine la manière dont différents établissements scolaires traduisent l'injonction politique contemporaine de faire de l'école un lieu d'éducation à la citoyenneté. La recherche conduit à formuler l'hypothèse du recul de la conception moderne de l'éducation à la citoyenneté, au profit d'un modèle à la fois plus pragmatique, plus ancré dans l'expérience, et concevant le bien commun comme un horizon à construire à partir des diversités. Ensuite, l'article fait état de trois traductions de la mission, faisant écho aux spécificités locales et aux identités organisationnelles des établissements.

\section{VEUGELERS Wiel, DE GROOT Isolde, STOLK Vincent, Research for CULT} Committee: Teaching common values in Europe, Bruxelles: Parlement européen, avril 2017, 214 p. [en ligne]

Cette étude explore l'enseignement des valeurs communes en Europe, en particulier la démocratie et la tolérance - si et comment elles font partie des programmes scolaires des États membres de l'Union européenne (UE) pour les élèves de l'enseignement secondaire. Le rôle de la société civile et des organisations non gouvernementales est également pris en considération. Les chapitres nationaux exposent la situation dans douze États membres de l'Union européenne et montrent que, dans de nombreux cas, il existe des écarts considérables entre les objectifs de la politique générale et l'application concrète des mesures concrètes d'application, ainsi qu'entre les politiques et les pratiques. https://bit.ly/2UrrR20 


\section{VALEURS ET DiVERSité CULTURELLE}

DE KETELE Jean-Marie (coord.), "Figures de l'éducation dans le monde. Dossier", Revue internationale d'éducation de Sèvres, décembre 2018, nº 79, p. 31-180 [en ligne]

Ce dossier est consacré aux grandes figures de l'éducation qui, au cours des siècles, ont fait évoluer l'éducation et ont eu une véritable influence dans l'histoire de l'éducation: de Confucius à Averroès et Ibn Khaldûn, de Dewey à Montessori, Vygotski ou Piaget, de Freire à Freud, Abreu, Condorcet, Rousseau ou Ferry, de la païdeia à l'évolution des conceptions de l'éducation en Afrique, un large éventail d'époques et de contextes est proposé.

https://journals.openedition.org/ries/6726

HALPERN Catherine, "La pensée morale à l'épreuve de la diversité", Sciences humaines, Grands dossiers n $n^{\circ}$, mars-avril-mai 2006 [en ligne]

D’une culture à l'autre, au sein d'une même société, voire pour chaque individu, différents principes éthiques peuvent se contredire. L'anthropologie et l'histoire, notamment, ont confronté l'homme des sociétés modernes occidentales à d'autres systèmes de valeurs. Les conflits moraux semblent inévitables. https://bit.ly/3yA5R3A

GAUTHIER Roger-François, ROBINE Florence, "Contenus et valeurs. Bigarrure du monde, convergence des questions", Revue internationale d'éducation de Sèvres, décembre 2009, $n^{\circ}$ 52, p. 77-84 [en ligne]

À partir de cinq études de cas (Angleterre, France, Inde, Sénégal, Suède), les auteurs constatent que la mondialisation n'entraîne pas d'uniformisation des contenus: cette pédagodiversité dans les valeurs enseignées est une richesse. En même temps, tous les systèmes sont confrontés à des questions semblables: les contenus vont de moins en moins de soi et sont devenus un élément majeur de questionnement sur la qualité de l'école, selon qu'ils sont plus ou moins cohérents et "pris au sérieux " par l'école elle-même.

https://journals.openedition.org/ries/744

KIM Yougsil, DUTERCQ Yves, "Le retour de l'éducation morale en Corée du Sud: un exemple d'éducationalisation ", Éducation et sociétés, 2018, n 42, p. 119-134

En Corée, il existe deux points de vue sur la question de l'éducation à la citoyenneté: si l'un se positionne en faveur de l'éducation morale, l'autre souligne la participation citoyenne. Cependant, en 2014, à la suite du naufrage d'un paquebot et du fait que les politiciens rejettent la responsabilité de la faute morale sur l'équipage, une loi sur l'éducation du caractère a été promulguée, qui encourage l'éducation morale. En l'absence de définition précise de l'éducation du caractère dans cette loi, chaque école organise les curriculums en fonction du sens empirique donné à cette notion. Certaines activités fondées sur cette éducation interviennent donc dans le domaine privé, usent d'une pédagogie reposant sur l'autorité et s'attachent aux valeurs traditionnelles.

MCNESS Elizabeth, "Les valeurs danoises, fondement de la Folkeskole", Revue internationale d'éducation de Sèvres, avril 2009, $n^{\circ}$ 50, p. 99-111 [en ligne]

L'article présente les valeurs ancestrales qui inspirent, encore aujourd'hui, les politiques éducatives, la structure et l'organisation des Folkeskolen danoises. Les valeurs de démocratie, de consensus, de collaboration et l'importance de la communication et des aspects sociaux et affectifs sont illustrées par le rôle clé du klasselorer, le professeur responsable de classe. https://journals.openedition.org/ries/522 
PARÉ-KABORÉ Afsata, "L'éducation traditionnelle et la vie communautaire en Afrique: repères et leçons d'expériences pour l'éducation au vivre-ensemble aujourd'hui ", McGill Journal of Education/Revue des sciences de l'éducation de McGill, 2013, vol. 48, $n^{\circ}$ 1, p. 15-33 [en ligne]

L'article identifie dans le mode de vie et d'éducation traditionnel en Afrique des pistes d'actions pour contribuer aux efforts d'organisation des systèmes scolaires afin de promouvoir l'éducation au vivre-ensemble aujourd'hui. La méthode employée est une approche comparative des contextes socio-éducatifs d'hier et de mutation d'aujourd'hui. Si l'on conçoit que l'éducation au vivre-ensemble peut être assumée par les systèmes éducatifs, ceux-ci doivent être conçus de manière holistique et être ouverts à une intervention des parents avec leurs outils davantage ancrés dans l'habitus communautaire. https://bit.ly/2V6qohR

\title{
WOLFF Francis, Plaidoyer pour l'universel, Paris: Fayard, 2019, 288 p.
}

Face à la montée des replis identitaires, des crispations religieuses et des idéologies nationalistes, l'auteur, spécialiste de philosophie ancienne, réhabilite l'universalisme, aussi bien dans le domaine de la rationalité scientifique que dans celui des valeurs morales. Reconnaissant l'origine humaine des valeurs, il plaide en faveur de l'humanisme et de l'aptitude au dialogue. Dans une première partie, l'auteur prend pour cible le relativisme culturel et se fait le défenseur d'une éthique humaniste et universaliste.

\section{LA QUESTION DES VALEURS EN FRANCE}

\begin{abstract}
ARKOUN Mohammed, AZEMA Jean-Pierre, BADINTER Élisabeth et al., Guide républicain. L'idée républicaine aujourd'hui, Paris: Canopé-CNDP/Ministère de l'éducation nationale, de l'enseignement supérieur et de la recherche/Delagrave, 2004, $430 \mathrm{p}$.
\end{abstract}

Deux parties principales composent ce guide: 1) un abécédaire des mots de la République rédigé par des intellectuels qui ont accepté de présenter de façon simple mais rigoureuse le sens de ces mots qui ont scandé l'histoire de France; 2) une anthologie regroupant 88 textes organisés en trois ensembles: voix d'hier, boussole pour aujourd'hui; questions et débats pour le temps présent; récits, poèmes et chansons. Des repères, des références complètent cet ouvrage.

BAUBEROT Jean, GAUCHET Marcel, OBIN Jean-Pierre, COQ Guy, "De l'école et des valeurs. Dossier", Administration et éducation, 2003, $n^{\circ} 100$, p. 63-131

Ce numéro consacré à l'école et ses valeurs rassemble plusieurs contributions qui éclairent les multiples aspects de cette question. Le premier article expose dans une perspective historique les valeurs morales qui sous-tendent l'institution scolaire et leur nécessaire évolution. Le deuxième article aborde d'un point de vue philosophique les questions d'égalité, d'autorité et de liberté de l'individu à l'école et montre l'intérêt de ces questions au sein de l'institution scolaire. Les trois autres contributions évoquent les valeurs transmissibles et les modalités de transmission de ces valeurs. 
BISSON-VAIVRE Claude, BOISSINOT Alain (coord.), "Laïcité, école et religions: dossier ", Administration et éducation, décembre 2015, no 148, p. 3-195 [disponible sur le portail Cairn]

L'institution scolaire est-elle encore à la hauteur de sa mission fondatrice, former le citoyen? La première partie propose deux tables rondes réunissant les témoignages d'élèves et d'enseignants: comment le débat sur les valeurs de la République pénètre-t-il dans les classes? Comment ces valeurs et leurs approches sont-elles perçues par les élèves euxmêmes? Les contributions suivantes s'organisent autour de deux grands pôles. Il s'agit d'abord de s'interroger sur ce que signifie, aujourd'hui, la référence à la laïcité et aux valeurs républicaines (liberté, égalité, fraternité). Le second pôle rassemble des articles qui tentent de réfléchir sur la place du religieux dans une école laïque. Plusieurs articles présentent la façon dont le débat est abordé au Québec, en Allemagne, au Japon et en Belgique.

Inspection générale de l'éducation nationale (IGEN), Valeurs de la République et laïcité. Séminaire des Doyens, Paris: Ministère de l'éducation nationale, de l'enseignement supérieur et de la recherche, avril 2015, 235 p. [en ligne]

Ce rapport est le fruit d'un travail collégial des groupes de l'IGEN conduit en avril 2015 sur les valeurs de la République et les responsabilités de l'école dans l'appropriation de ces valeurs ainsi que la façon dont elle les assume. Il met en lumière à quel point, au-delà des enseignements spécifiques et des différentes formes de mobilisations institutionnelles, cette mission première de l'école est assurée, dans le quotidien de la classe, par l'ensemble des disciplines et des spécialités. https://bit.ly/3wigt5S

OZOUF Mona, De Révolution en République. Les chemins de la France, Paris: Gallimard, 2015, 1376 .

Dans cet ouvrage, Mona Ozouf a rassemblé articles, conférences et chapitres de livres organisés en trois parties: "La Révolution », «La République », "La France, les France ». La première section reprend les principales recherches sur la Révolution française. Dans «Les idées et les mots ", l'autrice se concentre sur quelques idées au cœur du projet révolutionnaire, comme le principe du contrat social hérité de Rousseau ou l'idéal du « législateur pédagogue capable de faire surgir et de former une humanité neuve ». Mais c'est surtout l'école qui est le grand instrument de l'enracinement républicain sous la III République, et à laquelle un chapitre est dédié, école où a été donnée toute sa force à l'idée républicaine.

ROUX-LAFAY Corinne, "Comment nouer "le propre et le commun" dans l'enseignement de la morale à l'école?", Éthique en éducation et en formation, automne 2018, $n^{\circ}$ 5, p. 72-89 [en ligne]

En France, l'enseignement moral et civique (EMC), introduit par la loi de la refondation de l'école de 2013, vise à provoquer un questionnement sur les valeurs. Mais comment différencier les valeurs propres des valeurs communes configurant l'école de la République française, sinon en distinguant la morale d'inspiration déontologique et tendue vers un horizon d'universalité, de l'éthique qui relève d'un positionnement singulier? Cet article se propose de faire droit à deux axes de construction du sujet, l'un vertical, hérité de l'idéal laïc des Lumières, et l'autre traversé par la subjectivité. https://bit.ly/3xsw2J 